\title{
PASTAGEM DE AZEVÉM ASSOCIADA COM LEGUMINOSAS DE INVERNO SOB DIFERENTES NÍVEIS DE OFERTA DE FORRAGEM, NA REGIÃO SUL DO PARANÁ 1
}

\author{
RYEGRASS PASTURE ASSOCIETED WITH WINTER LEGUMES UNDER FOUR \\ LEVELS OF FORAGE ON OFFER, IN THE SOUTHERN REGION OF PARANÁ1
}

\author{
Oswaldo Teruyo IDO² \\ Anibal de MORAES ${ }^{3}$ \\ Adelino PELISSARI ${ }^{4}$ \\ Amir PISSAIA ${ }^{4}$ \\ Henrique Soares KOEHLER ${ }^{5}$
}

\begin{abstract}
RESUMO
Uma pastagem de azevém (Lolium multiflorum Lam.) associada com leguminosas de inverno: trevo vermelho (Trifolium pratense L.), trevo branco (Trifolium repens $L$.) e cornichão (Lotus corniculatus L.), submetida a quatro ofertas de forragem (OF), 4; 8; 12 e $16 \mathrm{~kg}$ de massa seca (MS) por $100 \mathrm{~kg}^{-1}$ de peso vivo (PV), foi avaliada num delineamento em blocos ao acaso, com duas repetições, no período de julho a novembro de 1995, no Centro de Estações Experimentais do Setor de Ciências Agrárias - UFPR Canguiri, em Pinhais-PR, com o objetivo de avaliar quatro níveis de oferta de forragem sobre a produção de pastagem de azevém associada a três leguminosas de inverno. O método de pastejo foi o contínuo com lotação variável, utilizando-se a técnica "put-andtake". Observou-se que a imposição de diferente OF condicionou diferentes quantidades de resíduo por unidade de área. Com o aumento na OF, aumentou gradativamente a MS residual ha-1. Maiores produções de MS ocorreram nas OF intermediárias. Na faixa de

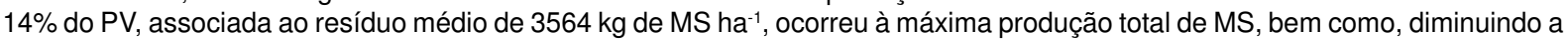
oferta de forragem aumentava a taxa de desaparecimento de MS.
\end{abstract}

Palavras-chave: Produção de massa seca; pastejo contínuo: Lolium multiflorum Lam., produção de forragem.

\begin{abstract}
A ryegrass pasture (Lolium multiflorum Lam.) associated with winter legumes: red clover (Trifolium pratense L.), white clover (Trifolium repens L.) and birdsfoot trefoil (Lotus corniculatus L.), submitted to four levels of herbage allowance (HA), namely 4; 8 ; $12 \mathrm{e} 16 \mathrm{~kg}$ of dry matter (DM) $100 \mathrm{~kg}^{-1}$ of the live weight (LW), was evaluated in a randomized complete block design with two replications. The trial was carried out in July until November 1995, at the Canguiri Experimental Station Center - SCA - UFPR, at Pinhais, PR with the objective of determinate four levels allowance herbage above production of Lolium multiflorum Lam. associated by three winter legumes. The "put-and-take" technique in a continuous grazing system with variable stocking rate was used. Different residuals dry matter/area was conditioned by the imposition of different HA. Increasing HA, caused gradual increase in residual DM ha' ${ }^{-1}$. The greatest DM production occurred in the intermediates $\mathrm{HA}$. Under the range of $14 \%$ of LW, associated with $3564 \mathrm{~kg}$ of residual DM ha-1, occurred maximum total DM production. Reducing herbage allowance increased the DM disappearance rate.

Key-words: Dry matter yield; continues grazing system; Lolium multiflorum; Forage yield.
\end{abstract}

\footnotetext{
${ }^{1}$ Parte da dissertação apresentada pelo primeiro autor à UFPR para obtenção do título de M.Sc.

${ }^{2} E_{n g}{ }^{\circ}$ Agr ${ }^{\circ}$ D.Sc., Prof. Adjunto do Depto. de Fitotecnia e Fitossanitarismo - Setor de Ciências Agraias da Universidade Federal do Paraná, caixa postal 19061, CEP 81531-990, Curitiba, PR. ; e-mail: ido@ufpr.br.

${ }^{3} E_{n g}{ }^{\circ}$ Agr $^{\circ}$ D.Sc., Prof. Adjunto, Orientador, Depto. de Fitotecnia e Fitossanitarismo - SCA/UFPR.

${ }^{4} E_{n g}^{\circ}$ Agro D.Sc., Prof. Adjunto, Co - Orientador, Depto. de Fitotecnia e Fitossanitarismo - SCA/UFPR

${ }^{5}$ Eng ${ }^{\circ}$ Florestal D.Sc., Prof. Adjunto, Co - Orientador, Depto. de Fitotecnia e Fitossanitarismo - SCA/UFPR.
} 


\section{INTRODUÇÃO}

As pastagens raramente estão em equilíbrio. $\mathrm{Na}$ maioria das vezes os animais consomem quantidade de forragem acima ou abaixo do que está sendo produzida. Alguma estabilidade do complexo solo - planta - animal é importante para a persistência de produção das pastagens. Isso só poderia ser conseguido através da combinação adequada dos fatores ambientais com aqueles controlados pelo homem: oferta de forragem, sistema de pastejo e tipo de animais utilizados (19). Destas, a oferta de forragem exerce papel fundamental no desempenho animal e na resposta da pastagem.

No Rio Grande do Sul, Quadros e Maraschin (18), em pastejo contínuo, observaram produções de $16,0 \mathrm{t} ; 12,3 \mathrm{t}$ e $10,8 \mathrm{t}$ de MS ha- ${ }^{-1}$ para as misturas de aveia preta (Avena strigosa Schreb) + azevém (Lolium multiflorum Lam.) + trevo vesiculoso (Trifolium vesiculosum Savi.); azevém + trevo vesiculoso e azevém + trevo branco + cornichão (Lotus corniculatus L.) respectivamente, em área adubada, submetida à oferta de forragem de 6 $\mathrm{kg}$ de MS $100 \mathrm{~kg}^{-1}$ de peso vivo (PV).

Trabalhos conduzidos a campo com pastejo contínuo em pastagem nativa, demonstram os efeitos de diferentes níveis de oferta de forragem $\left(4,8,12\right.$ e $16 \mathrm{~kg}$ de MS $100 \mathrm{~kg}^{-1}$ de PV dia-1). Escosteguy (5), observou que as maiores respostas foram obtidas em ofertas intermediárias, com a maior taxa de acúmulo de MS na OF de $12 \%$ do PV, enquanto Moojen (11) verificou um comportamento quadrático na mesma área experimental, com redução do crescimento nos maiores resíduos, na faixa de 11,5 a $13,6 \%$ do PV de OF obtiveram-se as maiores taxas de acúmulo da pastagem. Com novilhos em pastejo contínuo durante a estação de crescimento 1990/91, observaram uma relação positiva do resíduo para os níveis de oferta de forragem na primavera e no verão - outono (3).

Até o momento, no Paraná, a pesquisa de produção animal em pastagens cultivadas é irrelevante. Tanto no Paraná como em Santa Catarina, não se tem informações de pesquisa envolvendo produção animal com espécies de inverno, apesar da reconhecida importância do uso deste grupo de forrageiras na composição de sistemas de produção de carne e leite (14).

Para testar a hipótese: se a associação de pastagens de poáceas com fabáceas aumenta a oferta de forragem, então se pode melhorar o desempenho animal sob pastejo direto. O objetivo deste trabalho foi determinar o efeito de quatro níveis de oferta de forragem sobre a produção da pastagem de azevém associada com leguminosas de inverno, nas condições da Região Sul do Paraná.

\section{MATERIAL E MÉTODOS}

O experimento foi conduzido em um Latossolo Vermelho-Amarelo Álico, na Estação Experimental do Canguiri pertencente ao Setor de Ciências Agrárias da Universidade Federal do Paraná, no Município de Pinhais, Região Metropolitana de Curitiba, entre as coordenadas $25^{\circ} 25^{\prime}$ de latitude sul e $49^{\circ} 08^{\prime}$ de longitude oeste e com uma altitude de 915 metros. 0 clima, segundo a classificação de Köeppen é temperado do tipo Cfb (7).

A análise do solo da camada de 0 a $10 \mathrm{~cm}$ indicou os valores médios: $\mathrm{pH}(\mathrm{CaCl})=5,2 ; \mathrm{Al}^{+3}=0,02$ $\mathrm{cmol}^{(+)} \mathrm{dm}^{-3} ; \mathrm{Ca}^{2+}=7,6 \mathrm{cmol}^{(+)} \mathrm{dm}^{-3} ; \mathrm{Mg}^{2+}=7 \mathrm{cmol}^{(+)}$ $\mathrm{dm}^{-3} ; \mathrm{P}=6,8 \mathrm{mg} \mathrm{dm}^{-3} ; \mathrm{K}=0,14 \mathrm{cmol}^{(+)} \mathrm{dm}^{-3} ; \mathrm{C}=39 \mathrm{~g}$ $\mathrm{dm}^{3} ; \mathrm{V} \%=60$.

O plantio direto das forrageiras de inverno, com semeadora - adubadora de 15 linhas espaçadas de $17 \mathrm{~cm}$, foi realizada no período de 18 a 20 de abril de 1995 , com as seguinte quantidade de sementes por ha: $25 \mathrm{~kg}$ de azevém comum (Lolium multiflorum Lam.); $2 \mathrm{~kg}$ de trevo branco (Trifolium repens L.); $5 \mathrm{~kg}$ de trevo vermelho (Trifolium pratense L.); e $5 \mathrm{~kg}$ de cornichão (Lotus corniculatus L.), sendo as sementes das leguminosas previamente inoculadas com Rhizobium específicos, peletizadas, e misturadas com as do azevém. O controle das plantas daninhas e a dessecação da pastagem existente foram feitas através da aplicação de 2 litros ha ${ }^{-1}$ do herbicida comercial glyphosate, nos dias 04 e 05 de abril de 1995. Por meio de análises do solo e seguindo o cálculo pelo método da elevação da saturação de bases foi realizada a calagem a lanço, utilizando de 2 a 5 t ha ${ }^{-1}$ de calcário dolomítico, 12 dias de antecipação à semeadura. A adubação de manutenção foi realizada a lanço na semeadura, utilizando-se $60 \mathrm{~kg}$ de $\mathrm{P}_{2} \mathrm{O}_{5} \mathrm{e}$ $90 \mathrm{~kg}$ de $\mathrm{K}_{2} \mathrm{O}$ por ha. Aplicou-se em cobertura, $50 \mathrm{~kg}$ de $\mathrm{N} \mathrm{ha}^{-1}$ (uréia), parcelada em duas aplicações, 25 $\mathrm{kg}$. ha-1 de nitrogênio aos 27 dias e aos 72 dias após a semeadura.

Os tratamentos experimentais foram: quatro ofertas de forragem (OF) definidos como $\mathrm{kg}$ de massa seca (MS) por $100 \mathrm{~kg}^{-1}$ de peso vivo (PV) por dia. Os níveis foram de 4, 8, 12 e $16 \%$ do PV, dispostos em delineamento em blocos ao acaso com duas repetições por tratamento, totalizando 8 parcelas (potreiros), com áreas variando de 1,10 a 1,50 ha. As subdivisões foram feitas através de um sistema de cercas eletrificadas, mantendo-se um corredor interno para facilitar a movimentação dos animais até o centro de manejo e balança, bem como áreas adjacentes para manter os animais reguladores. O método de pastejo foi o contínuo, com lotação variável, utilizando a técnica dos animais reguladores "put - and - take" (16). Utilizou-se de dois animais "testers" (vacas em lactação da raça holandesa) previamente marcadas por tratamento. Foi usado número variável de animais reguladores (vacas secas e/ou novilhas) que eram manejadas de forma a manter os níveis desejados de oferta de forragem. O ajuste da carga animal 
(reguladores) foi realizada a cada 14 dias. Da semeadura ao início da utilização da pastagem transcorreram 89 dias. O período de utilização sob pastejo contínuo foi de 112 dias (17/07 a 06/11/95).

A estimativa do resíduo de (MS), da composição botânica e da freqüência das espécies da pastagem foi feita através da metodologia apresentada por Tothill et al. (20) denominada Botanal, acrescida das melhorias sugeridas por Jones e Hargreaves (9). As avaliações foram feitas antes do início do pastejo e posteriormente a cada 28 dias (período experimental). O Método do Disco (8) forneceu informações do resíduo de MS que eram utilizadas para o ajuste da carga animal nas diferentes ofertas de forragem a cada 14 dias.

A taxa de acúmulo, a taxa de desaparecimento e a produção de MS da pastagem foram determinadas a cada 28 dias, com o uso de três gaiolas de exclusão ao pastejo em cada potreiro, seguindo a técnica do triplo emparelhamento (15). O acúmulo total de MS no período experimental foi determinado a partir da taxa de acúmulo médio de MS multiplicado pelo número de dias do período avaliado, equivalente a 112 dias. A produção total de $\mathrm{MS}$ em $\mathrm{kg} \mathrm{ha}^{-1}$ fol determinada pela somatória das produções dos períodos (taxa de acumulação $\times \mathrm{n}^{\circ}$. de dias) mais 0 resíduo inicial estimado pelo método do disco, por ocasião da entrada dos animais. Para o cálculo da taxa de acúmulo de MS seca utilizou-se a equação descrita por Campbell (2). A estimativa de taxa de desaparecimento de MS seca foi determinada segundo a sistemática proposta por Moraes (13).

A oferta de forragem real, ou seja, a OF imposta de fato, foi calculada da seguinte forma: 1) adição do resíduo médio de $\mathrm{MS} \mathrm{ha}^{-1} \mathrm{com}$ a taxa de acúmulo estimada no período; 2) quociente deste valor pelo número de dias do período o que resultou no resíduo médio de MS estimado $\mathrm{ha}^{-1} \mathrm{dia}^{-1}$; 3) o quociente deste resultado, pela carga animal e 4) finalmente, chegou-se ao valor da OF real, pelo produto do valor obtido em três por 100, para a obtenção da porcentagem de oferta. Todos os animais eram pesados no momento da entrada e da saída do experimento e, a cada 28 dias. As pesagens eram realizadas logo após a ordenha da manhã. Para auxiliar no reajuste da carga animal foram necessárias as pesagens intermediárias (cada 14 dias).

A relação da variável independente OF com as seguintes variáveis dependentes: resíduo de MS ha $^{-1}$, taxa de acúmulo de MS, taxa de desaparecimento de MS, produção de MS, foi estabelecida através de análise de regressão. Procurou-se dentre os modelos, aquele que melhor ajustava a relação entre as variáveis, testando-se as regressões linear e quadrática. Cada um dos modelos foi submetido à análise de variância para determinar o nível de significância entre as variáveis. As análises foram feitas utilizando-se o manual simplificado de uso do programa MSTATC, elaborado por Koehler (10).

\section{RESULTADOS E DISCUSSÃO}

O período de avaliação da pastagem foi de julho a novembro de 1995, totalizando 112 dias. Os dados do resíduo médio de MS, avaliados em cada potreiro estão apresentados na Tabela 1. A partir do conhecimento da taxa de acúmulo de MS associado ao resíduo da pastagem, foi possível calcular as ofertas de forragem que definiram a OF real empregada.

TABELA 1 - Resíduos médios e ofertas de forragem avaliados na pastagem de azevém associada com leguminosas de inverno, utilizando o Método do Disco e o Método Botanal no período de 17/07/95 a 06/11/95. CEEx - Canguiri, SCA - UFPR. Pinhais-PR.

\begin{tabular}{cccccc}
\hline $\begin{array}{c}\text { OFERTA } \\
\text { PRETENDIDA }\end{array}$ & $\begin{array}{c}\text { POTREIRO } \\
(\% \mathrm{PV})\end{array}$ & \multicolumn{2}{c}{$\begin{array}{c}\text { RESÍDUO MÉDIO } \\
\mathrm{kg} \mathrm{MS} \mathrm{ha-1}\end{array}$} & \multicolumn{2}{c}{$\begin{array}{c}\text { OFERTA DE FORRAGEM REAL (c } \\
\text { PV) }\end{array}$} \\
\hline 4,0 & 02 & Disco & Botanal & Disco & Botanal \\
4,0 & 05 & 2073 & 1322 & 4,4 & 3,5 \\
8,0 & 04 & 2737 & 1436 & 4,7 & 3,8 \\
8,0 & 07 & 2949 & 2665 & 8,7 & 9,6 \\
12,0 & 03 & 3246 & 3550 & 9,6 & 13,8 \\
12,0 & 06 & 3321 & 3564 & 12,9 & 14,0 \\
16,0 & 01 & 3916 & 4107 & 13,3 & 19,2 \\
16,0 & 08 & 3925 & 4110 & 18,6 & 19,4 \\
\hline
\end{tabular}

Conforme a Tabela 2, conclui-se, que não existem diferenças significativas pelo teste "t" ao nível de $95 \%$ de probabilidade, entre as médias dos dados dos coeficientes de correlação, calculadas através de análise de regressão, obtidos pelo Método do Disco e pelo Método Botanal.
Para realizar o ajuste da carga animal nas diferentes ofertas de forragem a cada 14 dias, foram utilizados os valores dos resíduos de MS estimados através do Método do Disco, em razão deste método ser mais expedito. Entretanto, para teste do efeito dos tratamentos sobre as demais variáveis estudadas, optou-se pelas OF calculadas 
IDO, O.T. et al. Pastagem de azevém associada...

pelo Método Botanal, uma vez que, além dos dados de resíduos de $\mathrm{MS}$, a metodologia Botanal estimava também os demais parâmetros necessários: a composição botânica e a presença de todas as espécies na pastagem.

$\mathrm{Na}$ Tabela 3 podem ser vistos os dados referentes à taxa de acúmulo, taxa de desaparecimento e produção de MS.

TABELA 2 - Coeficientes de correlação obtidos em cada amostragem pelo Método do Disco e pelo Método Botanal. CEEx-Canguiri, SCA - UFPR. Pinhais-PR, 1995.

\begin{tabular}{ccc}
\hline DATA AMOSTRAGEM & MÉTODO DISCO & MÉTODO BOTANAL \\
\hline $11 / 07 / 95$ & 0.854 & 0.908 \\
$08 / 08 / 95$ & 0.969 & 0.968 \\
$05 / 09 / 95$ & 0.944 & 0.963 \\
$03 / 10 / 95$ & 0.953 & 0.916 \\
$06 / 11 / 95$ & 0.960 & 0.955 \\
\hline MÉDIA & 0.936 & 0.942 \\
\hline
\end{tabular}

$|t|=0,25 N S$

TABELA 3 - Taxas de acúmulo e de desaparecimento e produção total de MS na pastagem de azevém associada com leguminosas de inverno, submetida a diferentes ofertas de forragem, no período de 17/07 a 06/11/95. CEEx-Canguiri, SCA - UFPR, Pinhais - PR.

\begin{tabular}{cccccc}
\hline $\begin{array}{c}\text { OFERTA } \\
\text { REAL }\end{array}$ & POTREIRO & $\begin{array}{c}\text { TAXA } \\
\text { ACÚMULO }\end{array}$ & $\begin{array}{c}\text { TAXA } \\
\text { DESAPARECIMENTO. }\end{array}$ & $\begin{array}{c}\text { ACÚMULO } \\
\text { TOTAL MS NO } \\
\text { PERÍODO }\end{array}$ & $\begin{array}{c}\text { PRODUÇÄO } \\
\text { TOTAL MS }\end{array}$ \\
\hline \% PV & $\mathrm{N}^{\circ}$ & \multicolumn{2}{c}{$\mathrm{Kg} \mathrm{MS} \mathrm{ha}^{-1} \mathrm{dia}^{-1}$} & \multicolumn{2}{c}{$\mathrm{Kg} \mathrm{MS} \mathrm{ha}^{-1}$} \\
\hline 3,5 & 02 & 43,4 & 63,9 & 5861 & 7154 \\
3,8 & 05 & 46,5 & 71,8 & 5186 & 7816 \\
8,6 & 04 & 46,3 & 52,1 & 5365 & 8024 \\
9,2 & 07 & 47,9 & 59,5 & 5757 & 8993 \\
13,8 & 03 & 51,4 & 54,8 & 5880 & 9094 \\
14,0 & 06 & 52,5 & 58,1 & 5152 & 8481 \\
19,2 & 01 & 46,0 & 36,9 & 4995 & 8192 \\
19,4 & 08 & 44,6 & 30,7 & &
\end{tabular}

Pela Tabela 4, verifica-se que a variável, taxa de desaparecimento, apresentou relação significativa $(P<0,05)$ com a OF em ambos os modelos testados, sendo a regressão que apresentou o melhor ajuste entre as variáveis foi a linear. Com o aumento na OF, ocorreu redução progressiva na taxa de desaparecimento (Figura 1).

TABELA 4 - Relação linear e quadrática das variáveis analisadas no período de 17/07 a 06/11/95, com a oferta de forragem calculada, na pastagem de azevém associada com leguminosas de inverno. CEEx - Canguiri, SCA - UFPR, Pinhais-PR.

\begin{tabular}{|c|c|c|c|c|c|c|c|c|}
\hline & \multicolumn{4}{|c|}{ MODELO LINEAR } & \multicolumn{4}{|c|}{ MODELO QUADRÁTICO } \\
\hline VARIÁVEIS DEPENDENTES & $R^{2}$ & $\mathrm{~F}$ & $\mathrm{P}$ & C.V. \% & $\mathrm{R}^{2}$ & $\mathrm{~F}$ & $\mathrm{P}$ & C.V. \% \\
\hline Resíduo médio Botanal & 0,95 & 144,9 & $0,001^{\star}$ & 8,05 & 0,99 & 9631,4 & $0,001^{*}$ & 0,71 \\
\hline Taxa acúmulo MS & $-0,11$ & 0,30 & 0,604 & 7,04 & 0,49 & 4,3 & 0,081 & 4,78 \\
\hline Acúmulo Total MS & $-0,11$ & 0,30 & 0,605 & 7,05 & 0,49 & 4,3 & 0,080 & 4,78 \\
\hline Taxa desaparecimento & 0,76 & 22,8 & $0,003^{\star}$ & 12,55 & 0,78 & 13,1 & $0,010^{\star}$ & 12,05 \\
\hline Produção total MS & 0,33 & 4,42 & 0,080 & 6,24 & 0,72 & 9,9 & $0,018^{\star}$ & 4,03 \\
\hline Oferta Forragem Disco & 0,99 & 1457,4 & $0,001^{*}$ & 3,38 & 0,99 & 1149,3 & $0,001^{*}$ & 2,69 \\
\hline Resíduo médio Disco & 0,98 & 296,0 & $0,001^{*}$ & 3,83 & 0,98 & 203,1 & $0,001^{*}$ & 3,29 \\
\hline
\end{tabular}

OBS: $\quad$ * $(\mathrm{P} \leq 5 \%)$

$\mathrm{R}^{2}=$ Coeficiente de Determinação Ajustado; $\mathrm{F}=$ teste $\mathrm{F} ; \mathrm{P}=$ Probabilidade;

C.V. $=$ Coeficiente de Variação . 
Resposta semelhante foi encontrada por Moraes (13) em ensaio com pastagem de pangola associado à azevém + trevo branco, no período de inverno e de primavera, enquanto os autores (6) relatam que com ofertas maiores, a resposta do consumo torna-se progressivamente menor, até um ponto, acima do qual não existirá resposta de aumento de consumo com o aumento de forragem. Quando o pasto é intensamente pastejado, a forragem disponível tende a ser inteiramente consumida. Porém, sob pastejo leve, em condições de subpastejo, a ampla oferta de forragem permite que os animais pastejem seletivamente e ingiram quantidades máximas do alimento. Todavia, a oferta de forragem não deve ser muito alta, porque então o crescimento excessivo da pastagem permite acúmulo de forragem, que rapidamente perde o seu valor nutritivo por se tornar fibrosa, com baixos teores de proteínas (12).

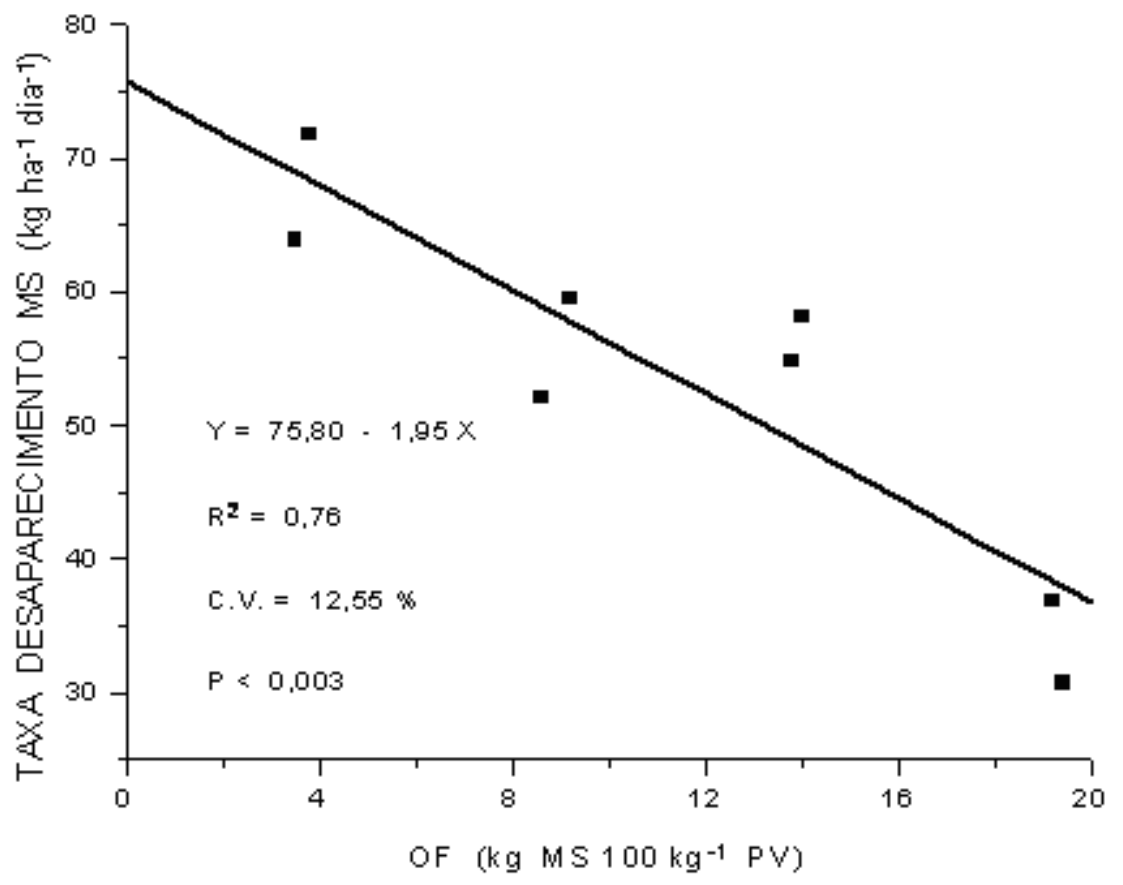

FIGURA 1 - Relação entre taxa de desaparecimento de MS com a oferta de forragem (OF) utilizada na pastagem de azevém, associada com leguminosas de inverno, avaliada no período de 17/07-06/11/95. CEEx-Canguiri, SCA/UFPR, Pinhais-PR.

De acordo com Blaser et al. (1), quando uma alta oferta de forragem está associada a muito material fibroso e velho (colmo ou material morto), o consumo de MS digestível e o desempenho animal decrescem. Além disso, pastagens sub utilizadas quando expostas à ação dos animais, estão sujeitas ao acamamento e ao desperdício, pelo excesso de material não consumido. A taxa de desaparecimento inclui, além da MS consumida pelos animais, o processo de senescência e toda a MS consumida por outros organismos presentes no meio, componentes da meso e micro fauna.

As taxas de acúmulo não tiveram um relacionamento significativo com as OF em ambos os modelos testados. Isto parece residir no fato de se dispor de apenas duas repetições por tratamento, o que reduz o número de graus de liberdade para o erro experimental, que é normalmente alto em experimento desta natureza (17). Outro fator que pode ter contribuído para não significância é o erro de amostragem, componente do erro experimental, havendo a possibilidade do número de amostras (3 gaiolas por potreiros) serem insuficientes. As amostragens, em experimentos de pastejo são feitas rente ao solo, o que inclui grande quantidade de material morto e senescente, que também deve ter concorrido para aumentar o erro. Para detectar possíveis diferenças nas taxas de acúmulo entre as diferentes OF, haveria necessidade de reduzir o período de corte nas gaiolas de exclusão e aumentar o número de gaiolas por potreiro (13).

Para o acúmulo total de MS no período experimental, pode-se dizer o mesmo em relação à taxa de acúmulo, uma vez que seu valor foi calculado pelo produto entre a taxa de acúmulo pelo número de dias do período avaliado, equivalente a 112 dias. Portanto, as mesmas considerações são válidas para o acúmulo total de MS. O acúmulo total de MS nos 
períodos avaliados no experimento (4860 a $5882 \mathrm{~kg}$ MS ha-1) foi equivalentes às obtidas por Moraes (13) no período de inverno - primavera (4375 a $5468 \mathrm{Kg}$ MS ha-1).

Conforme a Figura 2 houve significância $(P<0,018)$ para a relação entre a produção total de MS com a OF no modelo quadrático. Observa-se aumento gradativo na produção total de MS com o aumento da OF até um nível de OF intermediária. Acima deste, na maior OF, a produção total de MS começa a decrescer. Pelo uso do modelo ajustado, para a equação quadrática, foi estimado, que a máxima produção total de MS da pastagem durante o período experimental de 112 dias, corresponde a OF de 14,0\% do PV, com média de produção de $8783 \mathrm{~kg}$ de $\mathrm{MS} \mathrm{ha}^{-1}$, relacionado com o resíduo médio de $3564 \mathrm{~kg}$ de $\mathrm{MS} \mathrm{ha}^{-1}$. A produção total de MS de 9,09 t ha-1 (Tabela 3), considerando os resíduos iniciais estimado pelo método do disco, ficou abaixo das 10,86 t ha-1 encontradas por Quadros e Maraschin (18) para a mistura de azevém, trevo branco e cornichão, em condições de pastejo. Outros autores (4), também observaram produções ligeiramente superiores, variando de 10,32 a 10,89 t ha-1 de azevém anual.

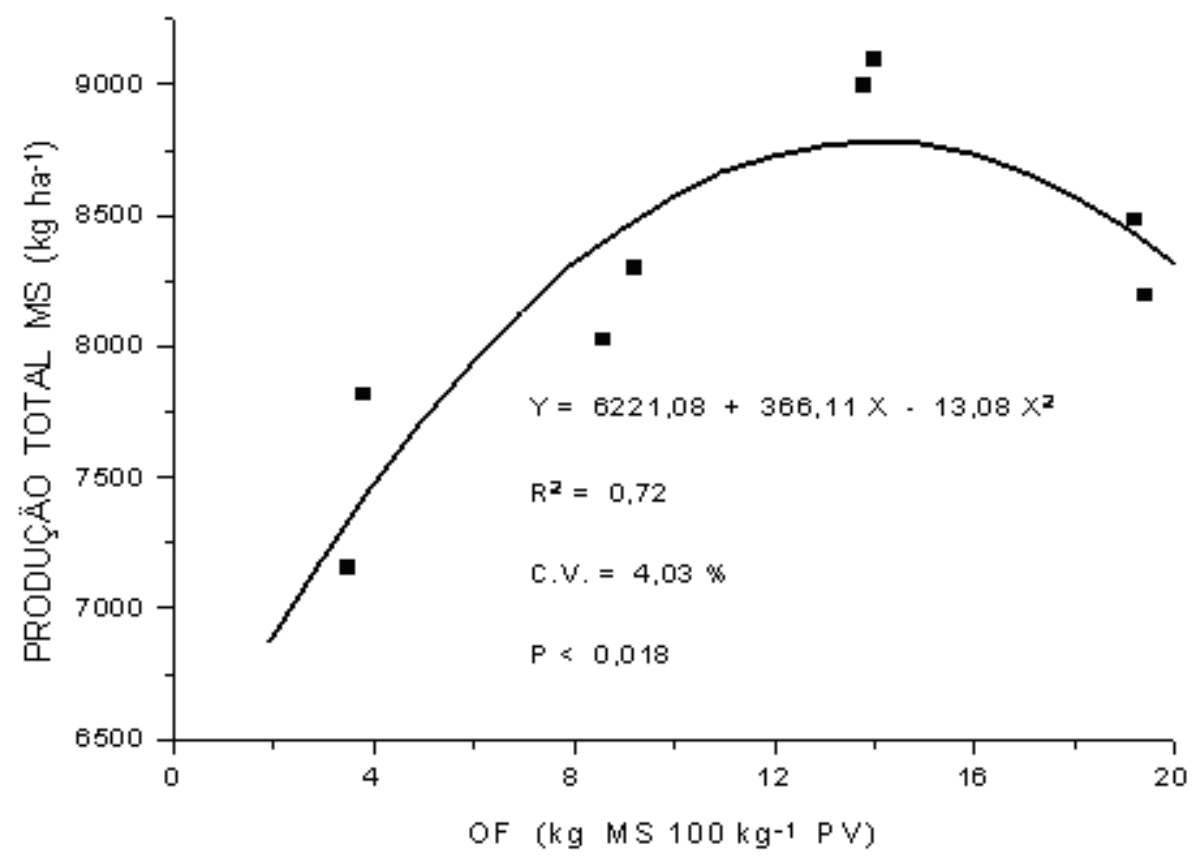

FIGURA 2 - Relação entre produção total de MS e a oferta de forragem (OF) utilizada na pastagem de azevém, associada com leguminosas de inverno, avaliada no período de 17/07 a 06/11/95. CEEx - Canguiri, UFPR - SCA, Pinhais - PR.

\section{CONCLUSÕES}

- A imposição de diferentes ofertas de forragem, condicionou diferentes quantidades de resíduo de MS por área;

- Com o aumento na oferta de forragem, há aumento gradativo na MS residual por unidade de área, resultando em maiores produções de MS seca nas ofertas intermediárias;

- Melhor aproveitamento da forragem pelos animais é indicado pelo aumento na taxa de desaparecimento de MS da pastagem associada à diminuição da oferta de forragem.

\section{REFERÊNCIAS BIBLIOGRÁFICAS}

1. BLASER, R.E.; HAMMES Jr., R. C.; FONTENOT, J.P.; et al. Forage-animal management systems. Virginia: Agricultural Experiment Station. Bulletin 86-7, 1986. 90p.

2. CAMPBELL, A.G. Grazed pastures parameters; I. Pasture dry-matter production and availability in a stocking rate and grazing management experiment with dairy cows. Journal of Agricultural Science, Cambridge, v. 67, p. 211-16, 1966.

3. CORRÊA, F.L.; MARASCHIN, G.E. Crescimento e desaparecimento de uma pastagem nativa sob diferentes níveis de oferta de forragem. Pesquisa. Agropecuária. Brasileira, Brasília, v.29, n.10, p.1617-1623, 1994. 
4. CULLETON, N.; MURPHY, W.E.; O'KEEFFE, W.F. The productivity of Lolium multiflorum Lam. under cutting and management. Journal of Agricultural Research, Wexford, v.26, p.105-113, 1987.

5. ESCOSTEGUY, C.M.D. Avaliação agronômica de uma pastagem natural sob níveis de pressão de pastejo. Porto Alegre, 1990. 231f. Dissertação (Mestrado em Zootecnia) -Faculdade de Agronomia, Universidade Federal do Rio Grande do Sul. 1990.

6. GREENHALGH, J.F.D.; REID, G.W.;AITKEN, J.N.; et al. The effects of grazing intensity on herbage consumption and production. I. Short term effects in strip-grazed dairy cows. Journal of Agricultural Science, Cambridge, n. 67, p.13-23, 1966.

7. INSTITUTO AGRONÔMICO DO PARANÁ. Cartas Climáticas do Estado do Paraná 1994. Londrina, PR., 1994. 49p. (IAPAR. Documento, 18).

8. JATEMBERG, W.D. Predicting the best time to apply nitrogen to grassland in spring. Journal of British Grassland Society, Hurley, v.25, p.266-71, 1970 .

9. JONES, R.M.; HARGREAVES, J.N.G. Improvements to the dry-weight-rank method for measuring botanical composition. Grass and Forage Science, Oxford, v.34, p.181-9, 1979.

10. KOEHLER, H.S. Manual de uso do programa MSTATC. Curitiba: UFPR/SCA-Departamento de Fitotecnia e Fitossanitarismo, 1996. 38p.

11. MOOJEN, E.L. Dinâmica e potencial produtivo de uma pastagem nativa do Rio Grande do Sul, submetida a pressões de pastejo, épocas de diferimento e níveis de adubação. Porto Alegre, 1991. 172p. Tese (Doutorado em Zootecnia)- Faculdade de Agronomia, Universidade Federal do Rio Grande do Sul. 1991.

12. MOORE, J.E.; MOTT, G.O. Structural inhibitors of quality in tropical grasses. In: ANTI-QUALITY COMPONENTS OF FORAGES. Madison: Crop Science Society of America, 1973. p.53-98

13. MORAES, A. de. Produtividade animal e dinâmica de uma pastagem de pangola (Digitaria decumbes Stent), azevém (Lolium multiflorum Lam.) e trevo branco (Trifolium repens L.), submetidas a diferentes pressões de pastejo. Porto Alegre, 1991. 200p. Tese (Doutorado em Zootecnia), Faculdade de Agronomia, Universidade Federal do Rio Grande do Sul. 1991.

14. MORAES, A. de.; MARASCHIN, G.E.; NABINGER, C. Pastagens nos ecossistemas de clima subtropical: Pesquisas para o desenvolvimento sustentável. In: SIMPOSIO SOBRE PASTAGENS NOS ECOSSISTEMAS BRASILEIROS: PESQUISAS PARA O DESENVOLVIMENTO SUSTENTÁVEL. (1995: Brasília). Anais. Brasília: Sociedade Brasileira de Zootecnia, 1995. p.146200.

15. MORAES, A. de.; MOOJEN, E.L.; MARASCHIN, G.E. Comparação de métodos de estimativa de taxas de crescimento em uma pastagem submetida a diferentes pressões de pastejo. In: REUNIÃO ANUAL DA SOCIEDADE BRASILEIRA DE ZOOTECNIA, (27: 1990: Campinas). Anais. Piracicaba: FEALQ, 1990. p.332.

16. MOTT, G.O.; LUCAS, H.L. The design, conduct and interpretation of grazing trials on cultivated and improved pastures. In: INTERNATIONAL GRASSLAND CONGRESS, (6.: 1952: Pensylvania). Proceedings. Pensylvania: State College Press, 1952. p.1380-1385.

17. PETERSEN, R.G.; LUCAS, H.L. Experimental errors in grazing trials. In: INTERNATIONAL GRASSLAND CONGRESS, (8.: 1960: Reading, England). Proceedings. Oxford: Alden Press, 1960. p.1380-5.

18. QUADROS, F.L.P.; MARASCHIN,G.E. Desempenho animal em misturas de espécies forrageiras de estaçao fria. Pesquisa Agropecuária Brasileira, Brasília, v.22 n.5, p.535-541, 1987.

19. SIMÃO NETO, M. Sistemas de pastejo 2. In: PASTAGENS: Fundamentos da exploração racional 2 ed., Piracicaba: FEALQ, 1994. p. 377-95, (Série atualização em Zootecnia, v.10).

20. TOTHILL, J.C.; HARGREAVES, J.N.G.; JONES,R.M. Botanal - a comprehensive sampling and computing method for estimating pasture yield and composition. I. Field sampling. Brisbane, CSIRO, Division of Tropical Crops and Pastures. 1978. 20p. (Tropical Agronomy Technical Memorandum, n.8). 